\title{
Extremal Problems under Dimension Constraints
}

\author{
R. Ahlswede, H. Aydinian, and L.H. Khachatrian \\ University of Bielefeld, Department of Mathematics, P.O. Box 100131, D-33501 \\ Bielefeld, Germany
}

\begin{abstract}
The aim of this paper is to systematically present an area of extremal problems under dimension constraints. We state conjectures and solutions for many of these problems. Proofs will be given in several papers, each devoted to a specific problem.
\end{abstract}

Key words: extremal algebraic theory, antichains, intersecting systems with weights, diametric, isoperimetric, and shadow problems, higher order incidence matrices, packing and covering.

\section{Introduction}

Often in life and in science we are going for extrema, maxima and minima. Historically, the first extremal problems which attracted many physicists/mathematicians were those in classical mechanics leading mainly from the $17^{\text {th }}$ to the $19^{\text {th }}$ century to an advancement of analysis, in particular the calculus of variation.

The $20^{\text {th }}$ century saw the uprising of Extremal Set Theory (or perhaps more generally Extremal Combinatorics), which primarily is concerned with finitely many objects and developped new methods which are mostly not analytic. Some are based on tools from algebra.

Even for problems not formulated in algebraic terms often in mathematics auxiliary algebraic structures are introduced to help in the analysis. Wellknown examples are Algebraic Topology and Algebraic Coding Theory.

This leads to extremal problems involving algebraic structures, in particular for linear spaces. In addition to Extremal Set Theory and Combinatorial 
Number Theory - essentially another child of the $20^{\text {th }}$ century - it makes therefore sense to term the new area Extremal Algebraic Theory.

Here we extend this theory by imposing dimension constraints on familiar set theoretic extremal problems.

We begin with some notation and definitions from extremal set theory.

Let $[n] \triangleq\{1, \ldots, n\}, 2^{[n]} \triangleq\{A: A \subseteq[n]\}$, and $\left(\begin{array}{c}{[n]} \\ w\end{array}\right) \triangleq\left\{A \in 2^{[n]}:|A|=\right.$ $w\}$. We associate with each subset $A$ its characteristic $(0,1)$-vector in $\mathbb{R}^{n}$. The corresponding notation for sets of $(0,1)$-vectors are: $E(n) \triangleq\{0,1\}^{n}$ and $E(n, w) \triangleq\left\{x^{n} \in E(n): x^{n}\right.$ has $w$ ones $\}$. The set theoretical notions like intersection, union, inclusion, antichain, etc. are extended to $(0,1)$-vectors in a natural way. The dimension of $\mathcal{S} \subset \mathbb{R}^{n}$ is defined by $\operatorname{dim}(\mathcal{S}) \triangleq \operatorname{dim} \operatorname{span}(\mathcal{S})$.

\section{A generic extremal problem under dimension constraint is the fol- lowing:}

Let $\mathcal{A} \subset E(n)$ satisfy some set-theoretical properties (say inclusion-free, pairwise non-empty intersections, etc.). In addition we require $\mathcal{A}$ to have $\operatorname{dim}(\mathcal{A})=k(k \leq n)$ and ask for the maximum (minimum) sized $\mathcal{A}$ with the given properties.

A basic question which naturally arises in this spirit is:

How many $(0,1)$-vectors can a $k$-dimensional subspace of $\mathbb{R}^{n}$ contain? The same question arises for $(0,1)$-vectors of given weight, that is, determine

$M(n, k, w) \triangleq \max \left\{\mid \mathcal{U} \cap E(n, w): \mathcal{U}\right.$ is a $k$-dimensional subspace of $\left.\mathbb{R}^{n}\right\}$.

The simple answer for the first question is $2^{k}$ and it is given in Odlyzko [24]. The second problem was considered first (for $k=n-1$ ) in Longstaff [19], where also an interesting application of this problem was presented. However $M(n, k, w)$ was determined (in [24]; see also [22]) only for $k=n-1$. Recently we gave the complete solution in [1].

Theorem AAK. (i) $M(n, k, w)=M(n, k, n-w)$.

$$
\text { (ii) } M(n, k, w)= \begin{cases}\left(\begin{array}{c}
k \\
w
\end{array}\right), & \text { if } 2 w<k \\
\left(\begin{array}{c}
2(k-w) \\
k-w
\end{array}\right) 2^{2 w-k}, & \text { if } k \leq 2 w \leq 2(k-1), 2 w \leq n \\
2^{k-1}, & \text { if } 2(k-1)<2 w \leq n\end{cases}
$$

In some cases it can be useful to consider a dual version of an extremal problem under dimension constraint. 
Dual problem (in terms of rank)

Given $M, n \in \mathbb{N}$ let $\mathcal{A}$ be an $M \times n(0,1)$-matrix having certain combinatorial properties. We ask now for the minimum $\operatorname{rank}(\mathcal{A})$ taken over all $M \times n$ matrices with those properties.

Thus the general problem can also be viewed as the problem of estimating the rank of $(0,1)$-matrices with certain combinatorial properties.

What are the motivations for studying the extremal problems under dimension constraints? Certainly among these motivations are connections to established areas. Let us mention some of them.

Combinatorial properties of $(0,1)$-matrices, in particular, investigation of minimum and maximum values of certain combinatorial parameters like rank, term rank, width, spectral radius, discrepancy, chromatic number etc.

Apparently Ryser was the first who started extensive investigations of combinatorial properties of $(0,1)$-matrices. For complete information on the problems and results in this subject see Brualdi and Ryser [8] (and also [9]).

With this connection let us mention an open problem, which was raised by Brualdi [9].

Let $N_{m, n}(r, \ell)$ be an $m \times n(0,1)$-matrix having $r$ ones in each row and $\ell$ ones in each column.

Problem. Determine or estimate the minimum possible rank of an $N_{m, n}(r, \ell)$ matrix.

For some partial results on this problem see [9], [10].

Note that if we remove the restriction on columns (or rows) then Theorem AAK gives the exact answer.

\section{Dimension or rank arguments}

Linear algebraic methods play an important role in extremal set theory. These have often been used to obtain very elegant, sometimes the only, proofs of many significant results (for interested readers we recommend the excellent forthcoming book by Babai and Frankl [7]). Among these methods and tools the dimension or rank arguments sometimes play a crucial role. The essence of dimension arguments is to associate with every member of a set system a vector from a properly chosen vector space and then to estimate the dimension of the spanned subspace, or the rank of a matrix associated with the set system.

The rank of an incidence matrix is an important parameter in many inci- 
dence structures like designs.

\section{Communication complexity}

In Yao's two-party communication complexity model [21] the rank of a communication $(0,1)$-matrix seems to play an important role. The relationship between deterministic communication complexity and matrix rank is one of the main research problems in this area (see [11], [23], [21]).

\section{Estimation of $(0,1)$-solutions of certain type of linear equations}

This question arises in several practical problems, for instance in problems of some database security models (see Griggs [18]).

Now we present our new Problems, Conjectures and Results.

\section{The Antichain Problem}

$\mathcal{F} \subset 2^{[n]}$ is called an antichain if $F_{1} \not \subset F_{2}$ holds for all $F_{1}, F_{2} \in \mathcal{F}$. One of the oldest results in extremal set theory is due to Sperner [26].

Theorem S. Let $\mathcal{F} \subset 2^{[n]}$ be an antichain, then

$$
|\mathcal{F}| \leq\left(\begin{array}{c}
n \\
\left\lfloor\frac{n}{2}\right\rfloor
\end{array}\right) .
$$

The maximum is assumed only for $\left(\begin{array}{c}{[n]} \\ \left\lfloor\frac{n}{2}\right\rfloor\end{array}\right),\left(\begin{array}{c}{[n]} \\ \left\lceil\frac{n}{2}\right\rceil\end{array}\right)$.

A result which implies the inequality in Theorem $\mathrm{S}$ is the well-known LYMinequality [13], [15].

Theorem LYM. Let $\mathcal{F} \subset 2^{[n]}$ be an antichain, then

$$
\sum_{F \in \mathcal{F}} \frac{1}{\left(\begin{array}{c}
n \\
|F|
\end{array}\right)} \leq 1
$$

The antichain problem under dimension constraint is to determine

$$
A(n, k) \triangleq \max \{|\mathcal{F}|: \mathcal{F} \subset E(n), \operatorname{dim}(\mathcal{F}) \leq k, \mathcal{F} \text { is an antichain }\}
$$

Conjecture 2.1. $A(n, k)=M\left(n, k,\left\lfloor\frac{n}{2}\right\rfloor\right)$. 
The next theorem of [2] partially establishes the conjecture.

\section{Theorem 2.1.}

(i) For $n \geq 2 k-2$

$$
A(n, k)=M\left(n, k,\left\lfloor\frac{n}{2}\right\rfloor\right)=2^{k-1} .
$$

(ii) $A(n, n-1)=M\left(n, n-1,\left\lfloor\frac{n}{2}\right\rfloor\right)= \begin{cases}2\left(\begin{array}{c}n-2 \\ \frac{n-2}{2}\end{array}\right), & \text { if } 2 \mid n \\ \left(\begin{array}{l}n-1 \\ \frac{n-1}{2}\end{array}\right), & \text { if } 2 \nmid n .\end{cases}$

\section{Intersection Problems}

We are given $1 \leq t \leq w \leq n, 1 \leq k \leq n$. A family $\mathcal{A} \subset 2^{[n]}$ is called intersecting (resp. $t$-intersecting) if $A_{1} \cap A_{2} \neq \varnothing$ (resp. $\left|A_{1} \cap A_{2}\right| \geq t$ ) holds for all $A_{1}, A_{2} \in \mathcal{A}$.

\section{The restricted case}

Intersection properties of set systems have been widely studied by many authors. The first result in this subject due to Erdős, Ko and Rado [14] was discovered by them in the late thirties, however, it was published more than 20 years later.

\section{Theorem EKR.}

(i) Let $\mathcal{F} \subset\left(\begin{array}{c}{[n]} \\ w\end{array}\right)$ be an intersecting family and $2 w \leq n$, then

$$
|\mathcal{F}| \leq\left(\begin{array}{c}
n-1 \\
w-1
\end{array}\right)
$$

(ii) Let $\mathcal{F} \subset\left(\begin{array}{c}{[n]} \\ w\end{array}\right)$ be t-intersecting, $w>t \geq 1, n \geq n_{0}(w, t)$, then

$$
|\mathcal{F}| \leq\left(\begin{array}{c}
n-t \\
w-t
\end{array}\right)
$$

For sharpenings of the EKR Theorem see [12], [16], [27]. The complete solution of the problem is given in [6]. Define now

$$
J_{t}(n, k, w) \triangleq \max \{|\mathcal{A}|: \mathcal{A} \subset E(n, w), \mathcal{A} \text { is } t \text {-intersecting, } \operatorname{dim}(\mathcal{A})=k\} .
$$

For the intersection problem with dimension constraints we believe the following 
Conjecture 3.1. For $w \leq \frac{n}{2}$

$$
J_{1}(n, k, w)=M(n-1, k, w-1) .
$$

Clearly, for $w>\frac{n}{2}$ one has

$$
J_{1}(n, k, w)=M(n, k, w)
$$

The next theorem confirms the conjecture for the case $w>\frac{k}{2}$.

Theorem $3.2[3]$. For $w \leq \frac{n}{2}$

$J_{1}(n, k, w)=M(n-1, k, w-1)= \begin{cases}2^{k-1}, & \text { if } k \leq w \leq \frac{n}{2} \\ 2^{2 k-2 w-2}\left(\begin{array}{c}2 k-2 w+2 \\ k-w+1\end{array}\right), & \text { if } k<2 w \leq 2(k-1) .\end{cases}$

The main auxiliary result which we used to prove this theorem is a LYMtype inequality for equations. Given $a_{1}, \ldots, a_{n}, \lambda \in \mathbb{R}^{+}$let $X \subset E(n)$ be the $(0,1)$-solutions of the equation

$$
\sum_{i=1}^{n} a_{i} x_{i}=\lambda
$$

Clearly $X$ corresponds to some antichain (whereas the opposite is not true). For a vector $v \in E(n)$ let $\|v\|$ denote the number of 1 's in $v$.

Lemma $3.3[3]$. Assume in (3.1) $a_{i} \neq a_{j}$ for some $i, j \in[n]$, and $\sum_{i=1}^{n} a_{i} \neq \lambda$. Then

$$
\sum_{x \in X} \frac{1}{\left(\begin{array}{c}
n \\
\|x\|
\end{array}\right)} \leq \frac{n-1}{n}
$$

As an important consequence (for our purposes) we get the following

Theorem 3.4 [3]. Let $f(n)$ be the maximum possible number of solutions of the equation (3.1) among all choices of $a_{1}, \ldots, a_{n}, \lambda \in \mathbb{R}^{+}$with $a_{1} \neq a_{2}$. Then

$$
f(n)= \begin{cases}2\left(\begin{array}{c}
n-1 \\
\frac{n-3}{2}
\end{array}\right), & \text { if } 2 \nmid n, n \geq 3 \\
\left(\begin{array}{c}
n \\
\frac{n-2}{2}
\end{array}\right), & \text { if } 2 \mid n\end{cases}
$$

In general the determination of $J_{t}(n, k, w)$ seems to be a difficult problem. However for big $k$ 's we have the following 
Theorem $3.5[3]$. For $1 \leq t \leq w$ and $k>k_{0}(w, t)$

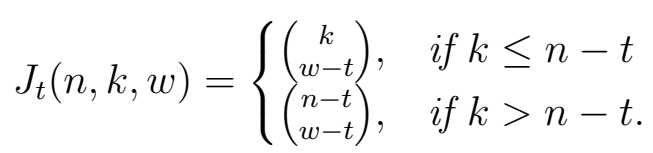

\section{The unrestricted case}

The following well-known result is due to Katona [19].

Theorem Ka. If $\mathcal{A} \subset 2^{[n]}$ is $t$-intersecting, $1 \leq t \leq n$, then

$$
|\mathcal{A}| \leq \begin{cases}|\mathcal{K}(n, t)|, & \text { if } 2 \mid(n+t) \\ 2|\mathcal{K}(n-1, t)|, & \text { if } 2 \nmid(n+t),\end{cases}
$$

where $\mathcal{K}(n, t)=\left\{A \in 2^{[n]}:|A| \geq \frac{n+t}{2}\right\}$ if $2 \mid(n+t)$.

Now with dimension constraint the problem is to determine

$$
J_{t}(n, k) \triangleq \max \{|\mathcal{A}|: \mathcal{A} \subset E(n), \mathcal{A} \text { is } t \text {-intersecting, } \operatorname{dim}(\mathcal{A})=k\} .
$$

Conjecture 3.6. For $t>n-k+1$

$J_{t}(n, k)= \begin{cases}|\mathcal{K}(k-1, t-(n-k+1))|+|\mathcal{K}(k-1, t+(n-k+1))|, & \text { if } 2 \mid(n+t) \\ 2|\mathcal{K}(k-2, t-(n-k+1))|+2|\mathcal{K}(k-2, t+(n-k+1))|, & \text { if } 2 \nmid(n+t) .\end{cases}$

Surprisingly this problem can be reduced to a weighted version of a $t$-intersection problem for set systems, formulated in a natural way.

\section{Reformulation of the problem}

Given $n, k \in \mathbb{N}$ we assign to each element $i \in[k]$ a weight $w_{i} \in \mathbb{N}$ such that $w_{i}+\cdots+w_{k}=n$. For any $F \in 2^{[k]}$ define the weight of $F$

$$
w(F)=\sum_{i \in F} w_{i}
$$

We say that a weighted set system $\mathcal{A} \subset 2^{[k]}$ is $t$-weight intersecting if $w\left(A_{1} \cap\right.$ $\left.A_{2}\right) \geq t$ for any $A_{1}, A_{2} \in \mathcal{A}$. Define the function

$$
f(n, k, t)=\max _{\sum_{i=1}^{k} w_{i}=n}\left\{|\mathcal{A}|: \mathcal{A} \subset 2^{[k]} \mathcal{A} \text { is } t \text {-weight intersecting }\right\} .
$$


Lemma $3.7[3]$.

$$
J_{t}(n, k)=f(n, k, t)
$$

We can explain now the sense of Conjecture 3 in terms of the function $f(n, k, t)$. The conjecture says that for a $t$-weight intersecting family $\mathcal{A} \subset 2^{[k]}$ we attain the maximum if $w_{1}=n-k+1, w_{2}=\cdots=w_{k}=1$, and

$$
\mathcal{A}= \begin{cases}\mathcal{K}(k, t)_{w} \triangleq\left\{A \in 2^{[k]}: w(A) \geq \frac{n+t}{2}\right\}, & \text { if } 2 \mid(n+t) \\ \mathcal{K}(k, t+1)_{w} \cup\left\{A \in 2^{[k-1]}: w(A)=\frac{n+t-1}{2}\right\} & \text { if } 2 \nmid(n+t) .\end{cases}
$$

Several results, partially proving the conjecture, were obtained in [3].

In particular we have the following

Theorem $3.8[3]$.

(i) For $t \leq n-k+1$

(ii) For $n \geq \frac{3}{2} k-1, t=n-k+2$

$$
J_{t}(n, k)=2^{k-1} .
$$

$$
J_{t}(n, k)=2^{k-2} .
$$

(iii) The conjecture holds for the cases

(a) $t \geq 2(n-k)-1$

(b) $k \leq n \leq k+3$

(c) $n \geq k \sqrt{2 k} / 2$.

\section{Diametric Problem}

Let us also mention the diametric problem for $(0,1)$-sequences of length $n$.

The Hamming distance between two vectors $\left(a_{1}, \ldots, a_{n}\right),\left(b_{1}, \ldots, b_{n}\right) \in E(n)$ is defined by $d_{H}(a, b)=\left|\left\{i \in[n]: a_{i} \neq b_{i}\right\}\right|$.

The diameter of a set $\mathcal{A} \subset E(n)$ is defined by $\operatorname{diam}(\mathcal{A}) \triangleq \max _{a, b \in \mathcal{A}} d_{H}(a, b)$.

Kleitman [20] proved the following

Theorem Kl. For a family $\mathcal{A} \subset E(n)$ with $\operatorname{diam}(\mathcal{A})=\delta<n$ one has

$$
|\mathcal{A}| \leq \begin{cases}\sum_{i=0}^{\delta / 2}\left(\begin{array}{c}
n \\
i
\end{array}\right), & \text { if } 2 \mid \delta \\
2 \sum_{i=0}^{(\delta-1) / 2}\left(\begin{array}{c}
n-1 \\
i
\end{array}\right), & \text { if } 2 \nmid \delta .\end{cases}
$$


It was shown in [5] that the intersection and diametric problems are equivalent, that is Theorem Ka and Theorem Kl can be reduced to each other.

Let us define now

$$
D_{k}(n, d)=\{|\mathcal{A}|: \mathcal{A} \subset E(n), \operatorname{diam}(\mathcal{A})=d, \operatorname{dim}(\mathcal{A}) \leq k<n\}
$$

Clearly if $k=n$ we have no dimension constraint, so Kleitman's Theorem gives the answer. The following simple observation shows that in all cases the diametric problem under dimension constraint reduces to Theorem Kl.

Theorem 4.1 [3].

$$
D_{k}(n, d)=D_{k}(k, d)
$$

Thus the intersection and diametric problems under dimension constraint are not equivalent!

\section{$5 \quad$ Forbidden weights in subspaces of $\mathbb{R}^{n}$}

The general problem is as follows.

Given a "forbidden" set $\mathcal{F} \subset E(n)$, let $\mathcal{U}$ be a $k$-dimensional affine subspace in $\mathbb{R}^{n}$ such that $\mathcal{U} \cap \mathcal{F}=\varnothing$.

Problem 1. (the unrestricted case)

Determine or estimate $\max _{\mathcal{U}}|\mathcal{U} \cap E(n)|$.

Problem 2. (the restricted case)

Determine or estimate $\max _{\mathcal{U}}|\mathcal{U} \cap E(n, m)|$.

In particular we consider the problem, when the forbidden set is $E(n, w)$ for some $1 \leq w \leq n$.

Then define

$$
F(n, k, w) \triangleq \max _{\mathcal{U}}\{|\mathcal{U} \cap E(n): \mathcal{U} \cap E(n, w)|=\varnothing\}
$$


The next result gives the complete answer for the case when $\mathcal{U}$ is a hyperplane. In this case $(k=n-1)$ we just write $F(n, w)$.

\section{Theorem $5.1[4]$.}

(i) $F(n, w)=F(n, n-w)$.

(ii) $F(n, w)= \begin{cases}\left(\begin{array}{c}2 w+1 \\ w+1\end{array}\right) 2^{n-2 w-1}, & \text { if } w<\frac{n}{2} \\ \left(\begin{array}{c}w \\ w\end{array}\right), & \text { if } w=\frac{n}{2} .\end{cases}$

Consider also the following problem.

Let $S$ be a hyperplane passing through the origin and define

$$
F S(n, w) \triangleq \max _{S}\{|S \cap E(n)|: S \cap E(n, w)=\varnothing\} .
$$

Surprisingly this problem turns out to be more difficult. For this case we have only some partial results [4].

(i) For $n=2 w, 2 w \pm 1,2 w \pm 2$ and $2 \nmid w$

$$
F S(n, w)=\left(\begin{array}{c}
n \\
\left\lfloor\frac{n}{2}\right\rfloor
\end{array}\right)
$$

(ii) $F S(n, n)=2^{n-1}$ (trivial)

(iii) $F S(n, 1)=\left(\begin{array}{c}n \\ \left\lfloor\frac{n}{2}\right\rfloor\end{array}\right)$

(iv) $F S(n, 3)=\left(\begin{array}{c}n \\ \left\lfloor\frac{n}{2}\right\rfloor\end{array}\right), n \geq 4$

(v) $F S(n, n-1)= \begin{cases}2^{n-2}, & \text { if } n \geq 9 \text { or } n=3,5,7 \\ \left(\begin{array}{c}n \\ \left\lfloor\frac{n}{2}\right\rfloor\end{array}\right), & \text { if } n=2,4,6,8 .\end{cases}$

For large n's we have

\section{Theorem $5.2[4]$.}

(i) Let $2 \mid w$, then for any $r \in \mathbb{N}$ and $n>n_{0}(r, w)$

$$
F S(n, w)<\left(\begin{array}{c}
n \\
\left\lfloor\frac{n}{2}\right\rfloor-r
\end{array}\right) .
$$

(ii) Let $2 \nmid w$, then for $n>n_{0}(w)$

$$
F S(n, w)=\left(\begin{array}{c}
n \\
\left\lfloor\frac{n}{2}\right\rfloor
\end{array}\right) .
$$

The simplest unsolved cases are $F S(n, 2)$ and $F S(n, n-2)$. Note that $F S(n, w) \neq$ $F S(n, n-w)$ unlike for $F(n, w)$. 
Conjecture 5.3. Let $n=3 \ell+r, 0 \leq r \leq 2$, then

$$
F S(n, 2)=2 \sum_{i=0}^{\ell}\left(\begin{array}{l}
\ell \\
i
\end{array}\right)\left(\begin{array}{c}
2 \ell+r-1 \\
2 i
\end{array}\right) \text {. }
$$

The corresponding $(n-1)$-dimensional subspace is defined by

$$
S=\left\{\left(x_{1}, \ldots, x_{n}\right) \in \mathbb{R}^{n}: 2 \sum_{i=r}^{\ell} x_{i}-\sum_{j=\ell+1}^{n-\ell-1} x_{j}=0\right\} .
$$

Conjecture 5.4. $F S(n, n-2)=11 \cdot 2^{n-6}, n \geq 6$.

The corresponding subspace is defined by

$$
S=\left\{\left(x_{1}, \ldots, x_{n}\right) \in \mathbb{R}^{n}: 2 x_{1}-x_{2}-x_{3}-x_{4}-x_{5}-x_{6}=0\right\} .
$$

Conjecture 5.5. For $2 \nmid w, w \leq \frac{n}{2}$

$$
F S(n, w)=\left(\begin{array}{c}
n \\
\left\lfloor\frac{n}{2}\right\rfloor
\end{array}\right) .
$$

Define also the "restricted" case of the same problem as follows.

$$
F S(n, w, m) \triangleq \max \{|\mathcal{B}|: \mathcal{B} \subset E(n, m), \operatorname{span}(\mathcal{B}) \cap E(n, w)=\varnothing\} .
$$

For this case we have the following

Theorem $5.6[4]$. Let $n=t m+r, 0 \leq r<m$.

(i) For $m \nmid w, m<w$, and $n>n_{0}(w, m)$

$$
F S(n, w, m)=t\left(\begin{array}{c}
n-t \\
m-1
\end{array}\right)
$$

(ii) For $w=s m$ and $n>n_{0}(w, m)$

$$
F S(n, w, m)=(s-1)\left(\begin{array}{c}
n-s+1 \\
m-1
\end{array}\right) .
$$

(iii) For $m>w$

$$
F S(n, w, m)=t\left(\begin{array}{c}
n-t \\
m-1
\end{array}\right) .
$$


The corresponding set $B \subset E(n, m)$ attaining this bound is $B=\left\{\left(b_{1}, \ldots, b_{n}\right) \in E(n, m): \sum_{i=1}^{t} b_{i}=1, \sum_{j=t+1}^{n} b_{j}=m-1\right\}$, for cases (i) and (iii) and $B=\left\{\left(b_{1}, \ldots, b_{n}\right) \in E(n, m): \sum_{i=1}^{s-1} b_{i}=1, \sum_{j=s}^{n} b_{j}=m-1\right\}$ for case (ii).

\section{Further Problems}

Let us state some further open problems of the same flavour which seem to be interesting.

The following two problems are related to a generalization of the function $M(n, k, w)$.

\section{$M(n, k, W)$-function}

Let $W=\left\{w_{1}, \ldots, w_{s}\right\} \subset[n]$ and let $E(n, W) \triangleq E\left(n, w_{1}\right) \cup \cdots \cup E\left(n, w_{s}\right)$. Define $M(n, k, W)=\max \{|\mathcal{A}|: \mathcal{A} \subset E(n, W), \operatorname{dim}(\mathcal{A})=k\}$.

\section{Higher order incidence matrices}

For integers $1 \leq \ell<m<n$ define the higher order incidence matrix $H(m, \ell)$ of size $\left(\begin{array}{c}n \\ m\end{array}\right) \times\left(\begin{array}{l}n \\ \ell\end{array}\right)$. The rows of $H(m, \ell)$ are labelled by $E \in\left(\begin{array}{c}{[k]} \\ m\end{array}\right)$ and the columns by $F \in\left(\begin{array}{c}{[n]} \\ \ell\end{array}\right)$, and the entry $h(E, F)$ is defined by

$$
h(E, F)= \begin{cases}1, & \text { if } F \subset E \\ 0, & \text { if } F \not \subset E .\end{cases}
$$

The problem is as follows. Given $1 \leq k \leq\left(\begin{array}{l}n \\ \ell\end{array}\right)$ determine or estimate the maximum number of rows of $H(m, \ell)$ such that these rows form a submatrix $M$ with $\operatorname{rank}(M)=k$.

This problem in its dual form was stated in Frankl and Tokushige [17].

Note that Theorem AAK gives the answer in the case $\ell=1$.

\section{$L$-systems under dimension constraints}

The following generalized intersection problem was raised by Sos [25]. 
Given $L=\left\{\ell_{1}, \ldots, \ell_{s}\right\} \subset[n]$ a family $\mathcal{A} \subset 2^{[n]}$ is called an $L$-system, if $\left|A_{1} \cap A_{2}\right| \in L$ holds for all distinct $A_{1}, A_{2} \in \mathcal{A}$.

The question is: how large can $|\mathcal{A}|$ be?

The same question can be asked for a uniform family $\mathcal{A} \subset\left(\begin{array}{c}{[n]} \\ w\end{array}\right)$.

There are many significant results in this direction (see for a good survey Frankl [15]).

The problem of $L$-systems under dimension constraints (the restricted and unrestricted cases) is formulated as for the case $L=\{t, \ldots, n\}$ ( $t$-intersecting systems).

\section{Shadow minimization problems}

The $\ell$-shadow of $\mathcal{A} \subset\left(\begin{array}{c}{[n]} \\ w\end{array}\right), 1 \leq \ell<k$, is defined by $\partial_{\ell} \mathcal{A}=\left\{F \in\left(\begin{array}{c}{[n]} \\ w-\ell\end{array}\right): \exists A \in \mathcal{A}: F \subset A\right\}$. Define also the colex order for the elements $A, B \in\left(\begin{array}{c}{[n]} \\ w\end{array}\right)$ as follows: $A<B \Leftrightarrow$ $\max ((A \backslash B) \cup(B \backslash A)) \in B$. We denote by $L_{m}(n, w)$ the initial $m$ members of $\left(\begin{array}{c}{[n]} \\ w\end{array}\right)$ in the colex order. The well-known Kruskal-Katona Theorem tells us how to minimize the $\ell$-shadow of a family with given size [13], [15].

Theorem KK. Let $\mathcal{A} \subset\left(\begin{array}{c}{[n]} \\ w\end{array}\right)$ with $|\mathcal{A}|=m$, then $\left|\partial_{\ell} \mathcal{A}\right| \geq\left|\partial_{\ell} L_{m}(n, w)\right|$.

The same problem can be considered with dimension constraint. That is, let $\mathcal{A} \subset E(n, w)$ with $\operatorname{dim}(\mathcal{A})=k$ and $|\mathcal{A}|=m \leq M(n, k, w)$, let $\partial_{\ell} \mathcal{A}$ be the $\ell$-shadow of $\mathcal{A}$ (defined in an obvious way), how small can $\left|\partial_{\ell} \mathcal{A}\right|$ be?

In the same way one can formulate

\section{Isoperimetric problems under dimension constraint}

For the formulations of isoperimetric type problems and results in this direction see Engel [13].

It seems also interesting to consider the problems discussed above for vector spaces over finite fields, in particular for $G F(2)^{n}$. Let us mention one of them: What is the analogue of $M(n, k, w)$ in $G F(2)^{n}$ ? Formally, determine or estimate

$m(n, k, w) \triangleq\left\{\mid \mathcal{U} \cap E(u, w): \mathcal{U}\right.$ is a $k$-dimensional subspace of $\left.G F(2)^{n}\right\}$.

This problem could be important from a coding theoretical viewpoint. We also found it interesting to consider the following type of problems.

\section{Packing and covering problems}


Let $\mathcal{A}_{1}, \ldots, \mathcal{A}_{m} \subset E(n)$ (resp. $\left.E(n, w)\right)$ and $\operatorname{dim}\left(\mathcal{A}_{i}\right)=k ; i=1, \ldots, m$. Then the system $\mathcal{A}_{1}, \ldots, \mathcal{A}_{m}$ is called a covering of $E(n)$ (resp. $\left.E(n, w)\right)$ if

$$
\bigcup_{i=1}^{m} \mathcal{A}_{i}=E(n) \quad\left(\operatorname{resp} . \bigcup_{i=1}^{m} \mathcal{A}_{i}=E(n, w)\right)
$$

The covering problem is to minimize $m$.

Let $\mathcal{A}_{1}, \ldots, \mathcal{A}_{m} \subset E(n)$ (resp. $\left.E(n, w)\right)$ such that $\operatorname{dim}\left(\mathcal{A}_{i}\right)=k$ and $\mathcal{A}_{i} \quad(i=$ $1, \ldots, m)$ are maximal sets, that is $\left|\mathcal{A}_{i}\right|=2^{k}$ (resp. $\left.\left|\mathcal{A}_{i}\right|=M(n, k, w)\right)$. Then the system $\mathcal{A}_{1}, \ldots, \mathcal{A}_{m}$ is called a packing of $E(n)$ (resp. $\left.E(n, w)\right)$ if for $i \neq j$ holds $\mathcal{A}_{i} \cap \mathcal{A}_{j}=\left\{0^{n}\right\}$ (resp. $\left.\mathcal{A}_{i} \cap \mathcal{A}_{j}=\varnothing\right)$.

The packing problem is to maximize $m$.

In particular we ask: Are there perfect packings (or tilings) of $E(n)$ or $E(n, w)$, i.e. packings which are simultaneously coverings? 


\section{References}

[1 ] R. Ahlswede, H. Aydinian and L.H. Khachatrian, Maximal number of constant weight vertices of the unit $n$-cube containing in a $k$-dimensional subspace, to appear in Combinatorica, special issue dedicated to the memory of P. Erdős.

[2 ] - Extremal problems under dimension constraints, Preprint 00-116, SFB 343 "Diskrete Strukturen in der Mathematik", Universität Bielefeld, 2000.

[3 ] - Intersection theorems under dimension constraints, Preprint 00-132, SFB 343 "Diskrete Strukturen in der Mathematik", Universität Bielefeld, 2000, submitted to J. Comb. Theory A.

[4] - Forbidden $(0,1)$-vectors in subspaces in $\mathbb{R}^{n}$, Designs, Codes and Cryptography, 29, 17-28, 2003.

[5 ] R. Ahlswede and G.O.H. Katona, Contributions to the geometry of Hamming Spaces, Discrete Math. 17, 1-22, 1977.

[6 ] R. Ahlswede and L.H. Khachatrian, The complete intersection theorem for systems of finite sets, Europ. J. Comb., 18, 125-136, 1997.

[7 ] L. Babai and P. Frankl, Linear algebra methods in combinatorics, to appear.

[8 ] R.A. Brualdi and H.J. Ryser, Combinatorial matrix theory, Cambridge Univ. Pr. 1992.

[9 ] R.A. Brualdi, Matrices of zeros of ones with fixed row and column sum vectors, Linear Algebra Appl. 33, 159-231, 1980.

[10 ] R.A. Brualdi, R. Mauber and J.A. Ross, On the minimum rank of regular classes of matrices of zeros and ones, J. Comb. Th. Series A 41, 32-49, 1986.

[11 ] B. Codenotti, G. Del Corso and G. Manzini, Matrix rank and communication complexity, Linear Algebra Appl., 304, 193-200, 2000.

[12 ] M. Deza and P. Frankl, Erdős-Ko-Rado Theorem - 22 years later, SIAM J. Algebra Discr. Math., 4, 419-431, 1983.

[13 ] K. Engel, Sperner Theory, Cambridge University Press, 1997.

[14 ] P. Erdős, Chao Ko and R. Rado, Intersection theorems for systems of finite sets, Quart. J. Math. Oxford 12, 313-320, 1961.

[15 ] P. Frankl, Extremal set systems, In Handbook of combinatorics, Edited by R. Graham, M. Grötschel and L. Lovász, ch. 24, 1295-1326, 1995.

[16 ] P. Frankl and Z. Füredi, Beyond the Erdős-Ko-Rado Theorem, J. Combin. Th. Ser. A 56, 182-194, 1991.

[17 ] P. Frankl and N. Tokushige, The Kruskal-Katona theorem, some of its analogues and applications, Extremal problems for finite sets, Bolyai Math. Soc., Budapest, 1994.

[18 ] J.R. Griggs, Database security and the distribution of subset sums in $\mathbb{R}^{m}$, Graph Theory and Combinatorial biology, Balatonelle 1996, Bolyai Math. Studs. 7, 223-252, 1999.

[19 ] G.O.H. Katona, Intersection theorems for systems of finite sets, Acta 
Math. Acad. Sci. Hungar., 15, 329-337, 1964.

[20 ] D.J. Kleitman, On a combinatorial conjecture of Erdős, J. Comb. Th. 1, 209-214, 1966.

[21 ] E. Kushilevitz and N. Nisan, Communication complexity, Cambridge Univ. Pr., 1997.

[22 ] W.E. Longstaff, Combinatorial solution of certain systems of linear equations involving (0,1)-matrices, J. Austral. Math. Soc. 23 (Series A), 266$274,1977$.

[23 ] N. Nisan and A. Wigderson, On rank vs. comunication complexity, Comb. 15(4), 557-665, 1995.

[24 ] A.M. Odlyzko, On the ranks of some $(0,1)$-matrices with constant row sums, J. Austral. Math. Soc. 31 (Series A), 193-201, 1981.

[25 ] V.T. Sós, Remarks on the connection of graphs, finite geometry and block designs, Colloq. Inter. Sulle Theorie Comb. (Rome 1973), Tomo II, Accad. Naz. Lincei, Rome, 223-233, 1976.

[26 ] E. Sperner, Ein Satz über Untermengen einer endlichen Menge, Math. Z. 27, 544-548, 1928.

[27 ] R.M. Wilson, The exact bound on the Erdős-Ko-Rado Theorem, Combinatorica, 4, 247-257, 1984. 